\title{
Effects of hot electron inertia on electron-acoustic solitons and double layers
}

\author{
Frank Verheest ${ }^{1,2, a)}$ and Manfred A. Hellberg ${ }^{2, b)}$ \\ ${ }^{1}$ Sterrenkundig Observatorium, Universiteit Gent, Krijgslaan 281, B-9000 Gent, Belgium \\ ${ }_{2}^{2}$ School of Chemistry and Physics, University of KwaZulu-Natal, Durban 4000, South Africa
}

(Received 27 March 2015; accepted 19 June 2015; published online 13 July 2015)

The propagation of arbitrary amplitude electron-acoustic solitons and double layers is investigated in a plasma containing cold positive ions, cool adiabatic and hot isothermal electrons, with the retention of full inertial effects for all species. For analytical tractability, the resulting Sagdeev pseudopotential is expressed in terms of the hot electron density, rather than the electrostatic potential. The existence domains for Mach numbers and hot electron densities clearly show that both rarefactive and compressive solitons can exist. Soliton limitations come from the cool electron sonic point, followed by the hot electron sonic point, until a range of rarefactive double layers occurs. Increasing the relative cool electron density further yields a switch to compressive double layers, which ends when the model assumptions break down. These qualitative results are but little influenced by variations in compositional parameters. A comparison with a Boltzmann distribution for the hot electrons shows that only the cool electron sonic point limit remains, giving higher maximum Mach numbers but similar densities, and a restricted range in relative hot electron density before the model assumptions are exceeded. The Boltzmann distribution can reproduce neither the double layer solutions nor the switch in rarefactive/compressive character or negative/positive polarity. (C) 2015 AIP Publishing LLC. [http://dx.doi.org/10.1063/1.4926513]

\section{INTRODUCTION}

Nonlinear electrostatic waves in plasmas were first described by a Korteweg-de Vries (KdV) equation, ${ }^{1}$ through a reductive perturbation analysis, with nonlinear ion-acoustic modes as the simplest and earliest example. ${ }^{2}$ A great many papers have attested to the fact that other modes and plasma compositions could also be handled, as long as the nonlinearities were not too large. However, this restriction was soon lifted by describing solitary waves of arbitrarily large amplitudes within the framework of the Sagdeev pseudopotential analysis, ${ }^{3}$ in a frame which comoves with the solitary wave, for one mode at a time. By a generally sanctioned abuse of notation, such large-amplitude solitary waves are often referred to as "solitons," even though the Sagdeev formalism excludes all discussion of their stability and interaction properties.

Acoustic-type electrostatic modes require at least one species whose thermal velocity is smaller than the structure velocity and also at least one species with a larger thermal velocity. ${ }^{4,5}$ In the simplest example of the ion-acoustic soliton, in a proton-electron plasma, the heavier ions are described as a cold fluid (their temperature effects neglected), while the hot electrons are treated as being Boltzmann-distributed (that is, their inertia is neglected). The latter simplification, however, assumes explicitly or implicitly that the soliton structure speed is considerably smaller than the hot electron thermal velocity. Such a requirement is fundamental for the validity of the results, yet very difficult to check a posteriori or quantify, as the latter

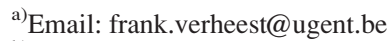

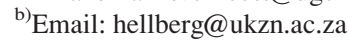

velocity has, of course, been assumed to be infinite by use of the Boltzmann distribution. More modern approaches, treating the hot species distributions as deviating from a Maxwellian, like the Cairns, ${ }^{6} \mathrm{kappa},{ }^{7,8}$ or Tsallis ${ }^{9,10}$ distributions, also neglect hot species inertia, and are thus subject to the same caveat.

There have been rather sporadic discussions in which authors have tried to compare the inertialess and the inertial treatments, so as to gain a better feeling for how reliable results derived in an inertialess approach are, and which restrictions on the parameter ranges might follow from such a comparison. Some of these relate to the effects of heavy species' drifts on acoustic soliton behaviour. An early exam$\mathrm{pl}^{11}$ used a KdV approach in comparing ion relativistic effects and electron finite-mass effects, and reported that the latter were considerably larger. Some other more recent papers involving small amplitude expansions, include, for instance, inertial isothermal light species in studying dustacoustic solitons, ${ }^{12}$ and effects of viscosity ${ }^{13}$ or isothermal electrons and warm dust ${ }^{14}$ on dust-ion-acoustic solitons. However, none of these have carried out systematic arbitrary amplitude investigations of the effects of considering the light species as being inertial and isothermal rather than inertialess.

Following on the work of Kuehl and Zhang, ${ }^{11}$ Rice et al. ${ }^{15}$ used the Sagdeev approach to consider finite electron mass effects on ion-acoustic solitons in a two electron temperature plasma, while Mace and Hellberg ${ }^{16}$ investigated an analogous model related to dust-acoustic double layers in a three-species dusty plasma with two positive ion temperatures. Although finite electron inertia was shown to have a negligible effect on positive potential ion-acoustic solitons, 
large reductions in the maximum amplitudes and the existence domains of negative potential ("rarefactive") solitons and double layers were found. ${ }^{15}$ Analogous effects (but with reversed polarity) were reported by Mace and Hellberg ${ }^{16}$ for dust-acoustic double layers. It was shown that these large effects were due to a break down of the underpinning model when amplitudes exceeded a critical value. This was related to the ratio of the nonlinear structure speed to the thermal speed of the cooler of the two "hot" components. Where the latter component is modeled by an inertialess expression, such as the Boltzmann relation, this limit of the model is ignored, as the thermal speed is assumed to be infinite.

Later, this work was followed by Cattaert et al. ${ }^{17}$ and Verheest et al., ${ }^{18}$ who used the McKenzie fluid dynamical approach $^{4,19}$ (a fully nonlinear technique that is analogous to the Sagdeev formalism), in investigating electron-acoustic solitons and double layers. They showed that both positive and negative potential electron-acoustic structures could exist in a plasma in which there were no drifts. This was in contrast to general beliefs at the time, in that a number of papers had considered the effects of drifts in attempts to explain space observations of both polarities of nonlinear electron-acoustic structures. ${ }^{20,21}$

However, honesty forces one to admit that the above efforts at systematic studies have not resonated much nor do they boast of a great following, and that the large majority of papers still use one or other inertialess description for the hot species.

In this paper, we investigate nonlinear electron-acoustic modes in a plasma composed of positive ions and a twotemperature electron distribution. There are three thermal velocities in the system, ordered in the usual way from low to high, of the ions, the cool electrons, and the hot electrons. In such a three-component plasma, there are two overall acoustic velocities, a lower one, in the window between the ion and the cool electron thermal velocities, and a higher one, in the window between the cool and the hot electron thermal velocities. There is a further range with phase speeds greater than the hot electron thermal speed. However, that does not yield an acoustic mode, but the electron plasma wave in a two-electron plasma.

Before going on, we would like to point out that one can compute these acoustic speeds in two fully equivalent ways, either from the electrostatic dispersion law for linear harmonic waves in the long-wavelength limit or from the proper convexity condition of the pseudopotential in a Sagdeev approach for nonlinear solitary waves. Because both methods yield precisely the same values ${ }^{4,5,22-24}$ and we are interested in the nonlinear regime, there is no need to treat first the linear modes.

The lower acoustic mode is the ion-acoustic wave, with the ions providing the inertia, and the cool and hot electrons the pressure to sustain the wave. The higher acoustic mode is usually called electron-acoustic, since it is on the electron timescale, with a phase speed lying between the cool and hot electron thermal speeds. Hence, the ions can play only a minimal role, with the two electron components providing both the pressure and the inertia to sustain the wave. This will be further illustrated in Sec. II, when the analytical convexity conditions are derived.

In the literature, one encounters several simplifications in the description of some of the species. On the lower side, the ions can be strictly cold, or cool enough to respect the ordering of the thermal velocities, or may even be treated as an immobile, neutralizing background in the electronacoustic regime. On the other hand, the hot electrons are, much more often than not, treated as inertialess, through Boltzmann or more sophisticated distributions, the latter reflecting various forms of nonthermal behaviour at the phase space level.

The model that we have chosen keeps the ion dynamics on board, but treats the ions as strictly cold, to simplify the algebra and also because the ion thermal effects obviously play a very subsidiary role in the electron-acoustic mode. The cool electrons are described through an adiabatic pressure-density relation, and their inertia is essential to respect the electron-acoustic ordering. We have chosen to retain also the inertial effects of the hot electrons, in order to make a valid comparison with the inertialess description, which dominates in the literature. As the thermal speed of the hot electrons is much greater than the phase speed and the typical soliton speeds, one may assume that heat flow is possible, and hence they behave isothermally (with $\gamma=1$ ). This model reduces to the commonly used inertial Boltzmann distribution for infinite thermal speed.

In contrast to the above discussion, we note that there have been some studies of ion-acoustic and electron-acoustic solitons in plasmas in which all species are assumed to be adiabatic. $^{25-28}$ Thus, even the species having the highest thermal speeds are assumed to behave adiabatically. Like the isothermal hot electron model, the adiabatic model takes account of the effects of finite hot electron inertia. However, it also changes significantly the kind of physical response that is assumed for the hot species from that underpinning the Boltzmann approximation, thus making an investigation of the inertial effects alone more difficult.

Furthermore, the assumption of adiabatic hot electrons implies that they appear to be thermally insulated, with thermal conduction being slow. That is, their thermal speed is at most only marginally greater than the wave speed. Although there are probably situations where such a model is valid, it would seem that it is subject to significant restrictions on the range of hot electron thermal speeds that fit it. We recall that for thermal speeds sufficiently close to the phase speed, there will be linear Landau damping, thus potentially preventing sustainable nonlinear structures from being generated. Unfortunately, the papers do not appear to have quantified the range of thermal speeds that would be permitted by the physics on which the fully adiabatic model is based.

Given that the extant literature on electron-acoustic modes is vast, and that we are focusing on hot electron inertia effects in the nonlinear regime, we have only quoted references in which there has been a genuine effort to assess what the effects of retaining finite mass effects for the hot electrons are on arbitrary amplitude structures, as compared to the usual assumptions that for the hottest species inertial effects can be neglected. 
As we shall see in what follows below, retaining inertia in the calculation by considering hot isothermal electrons yields a switch in polarity in certain parameter ranges, a result that is in line with earlier investigations along these lines. ${ }^{17}$ At the same time, switching between inertial and inertialess models shows that the inertialess (Boltzmann) description is only valid for a fairly small range in relative cool electron density.

The paper is structured as follows: the model equations are briefly recalled in Sec. II, Sec. III then containing the analysis and discussion. We summarize our conclusions in Sec. IV.

\section{MODEL EQUATIONS}

The model we study here comprises singly-charged, cold positive ions (label $i$ ), cool adiabatic (label $c$ ) and hot isothermal (label $h$ ) electrons. The assumption of singly-charged ions is not a restriction, as one can easily deal with multiplycharged ions by modifying the normalization. The fraction of negative charge residing on the cool electrons is $f=n_{c 0} / n_{i 0}$ and on the hot electrons $1-f=n_{h 0} / n_{i 0}$, in terms of the positive ion density $n_{i 0}$. The normalization has been carried out with respect to the cool electron parameters: a mass ratio $\mu=m_{e} / m_{i}$, a kind of Mach number $M=V / c_{t c}$ (where $V$ is the speed of the solitary wave structure with respect to an inertial frame and $c_{t c}=\sqrt{T_{c} / m_{e}}$ is the cool electron thermal velocity), a kinetic temperature ratio $\sigma=T_{h} / T_{c}$ (in energy units), and the electrostatic potential $\varphi$ is normalized by $T_{c} / e$. For the electron acoustic mode, the ordering, $c_{t c}<V<c_{t h}$, is $1<M<\sqrt{\sigma}$ in normalized form.

The continuity equations for the three species, with running label $\alpha$, are

$$
\frac{\partial n_{\alpha}}{\partial t}+\frac{\partial}{\partial x}\left(n_{\alpha} u_{\alpha}\right)=0
$$

whereas the momentum equations are more distinct

$$
\begin{gathered}
\frac{\partial u_{i}}{\partial t}+u_{i} \frac{\partial u_{i}}{\partial x}+\mu \frac{\partial \varphi}{\partial x}=0, \\
\frac{\partial u_{c}}{\partial t}+u_{c} \frac{\partial u_{c}}{\partial x}+n_{c} \frac{\partial n_{c}}{\partial x}-\frac{\partial \varphi}{\partial x}=0, \\
\frac{\partial u_{h}}{\partial t}+u_{h} \frac{\partial u_{h}}{\partial x}+\frac{\sigma}{n_{h}} \frac{\partial n_{h}}{\partial x}-\frac{\partial \varphi}{\partial x}=0 .
\end{gathered}
$$

Here, $u_{\alpha}$ are the normalized species velocities, and we have used the pressure-density relation $p_{c}=n_{c}^{3}$ and $p_{h}=n_{h}$.

In a frame where the nonlinear structure is stationary $(\partial / \partial t=0)$ and all variables tend to their undisturbed values at $x \rightarrow-\infty$, in particular, $\varphi \rightarrow 0$, one integrates the ion and cool electron equations, (1)-(3) with respect to $x$ and obtains normalized densities

$$
\begin{gathered}
n_{i}=\left(1-\frac{2 \mu \varphi}{M^{2}}\right)^{-\frac{1}{2}}, \\
n_{c}=\frac{1}{2}\left[\sqrt{(M+1)^{2}+2 \varphi}-\sqrt{(M-1)^{2}+2 \varphi}\right] .
\end{gathered}
$$

The latter expression is easily found using standard methods. ${ }^{29,30}$

In order to bring out the differences between keeping and omitting the hot electron inertial effects, while treating the species as behaving isothermally, we proceed to the Bernoulli integrals ${ }^{4,19,31}$ by integrating (4) and using mass conservation from (1). This yields a relation between $n_{h}$ and $\varphi$

$$
\frac{M^{2}}{2}\left(\frac{1}{n_{h}^{2}}-1\right)+\sigma \ln n_{h}=\varphi .
$$

A similar equation for the density of an isothermal component was found in analogous ion-acoustic and dust-acoustic soliton studies. ${ }^{15,16} \mathrm{We}$ note here that the first term arises from including finite inertia. When hot electron inertia is neglected, the inversion of this integral to express $n_{h}$ as a function of $\varphi$ leads to the well known Boltzmann expression

$$
n_{h B}=\exp \left(\frac{\varphi}{\sigma}\right)
$$

where the subscript $B$ reminds us that we are using the Boltzmann expression. An important point is that the validity of (8) requires that $M \ll \sqrt{\sigma}$, which, as we shall see, is a severe restriction.

Retaining hot electron inertia leads in the usual Sagdeev approach, in which the pseudopotential is expressed in terms of the electrostatic potential, to the need for a numerical solution. ${ }^{15,16}$ Instead, we shall use (7) to express $\varphi$ in terms of $n_{h}$, also in the cold ion and cool electron densities. We will then subscript the hot electron density with $A, n_{h A}$, as this will be the main focus of the analytical treatment.

The densities are substituted into Poisson's equation

$$
\frac{d^{2} \varphi}{d x^{2}}+n_{i}-f n_{c}-(1-f) n_{h}=0,
$$

which has to be integrated to obtain an energy-like integral

$$
\frac{1}{2}\left(\frac{d \varphi}{d x}\right)^{2}+S(\varphi, M)=0
$$

in terms of a Sagdeev pseudopotential $S(\varphi, M)$.

However, this procedure only works if all three densities can be expressed in terms of $\varphi$, hence for the case of Boltzmann electrons. Using (5), (6), and (8) in (9) thus gives upon integration that

$$
\begin{aligned}
S_{B}(\varphi, M)= & \frac{M^{2}}{\mu}\left(1-\sqrt{1-\frac{2 \mu \varphi}{M^{2}}}\right) \\
& +\frac{f}{6}\left\{6 M^{2}+2-\left[(M+1)^{2}+2 \varphi\right]^{3 / 2}\right. \\
& \left.+\left[(M-1)^{2}+2 \varphi\right]^{3 / 2}\right\} \\
& +(1-f) \sigma\left[1-\exp \left(\frac{\varphi}{\sigma}\right)\right] .
\end{aligned}
$$

Here, as in the following, the purely compositional parameters $(f, \mu, \sigma)$ are not explicitly listed as arguments of $S(\varphi, M)$, so as not to overload the notation. The integration constants 
have been adjusted to ensure that $S_{B}(0, M)=0$, for the undisturbed conditions faraway from the solitary structure, which is standard practice in Sagdeev pseudopotential analysis.

When hot electron inertia is retained, we cannot invert (7) and instead express (5) and (6) in terms of $n_{h A}$ by defining $\varphi$ as a function of $n_{h A}$, given in (7). In this case, we will use $n=n_{h A}$ for notational brevity and write (7) in short as $\varphi=F(n)$. To keep it simple, we use the integrated forms of $n_{i}$ and $n_{c}$, obtained in (11), and then replace $\varphi$ by $F(n)$. We have to compute separately, however, the hot electron contribution

$$
\int n d \varphi=\int n\left(-\frac{M^{2}}{n^{3}}+\frac{\sigma}{n}\right) d n=M^{2}\left(\frac{1}{n}-1\right)+\sigma(n-1),
$$

where now the undisturbed conditions are at $n=1$.

The change from $\varphi$ to $n$ also has repercussions on the term in $d \varphi / d x=(d n / d x)(d \varphi / d n)$ in (10). Collecting all this together yields

$$
\frac{1}{2}\left(\frac{d n}{d x}\right)^{2}+S_{A}(n, M)=0
$$

in terms of a quite different, more complicated Sagdeev pseudopotential

$$
\begin{aligned}
S_{A}(n, M)= & \frac{n^{6}}{\left(M^{2}-\sigma n^{2}\right)^{2}}\left\{\frac{M^{2}}{\mu}\left(1-\sqrt{1-\frac{2 \mu F(n)}{M^{2}}}\right)\right. \\
& +(1-f)\left[M^{2}\left(1-\frac{1}{n}\right)+\sigma(1-n)\right] \\
& +\frac{f}{6}\left(6 M^{2}+2-\left[(M+1)^{2}+2 F(n)\right]^{3 / 2}\right. \\
& \left.\left.+\left[(M-1)^{2}+2 F(n)\right]^{3 / 2}\right)\right\}
\end{aligned}
$$

For further comparison between the inertial and Boltzmann approach to the hot electron characteristics, we cannot use $S_{B}(\varphi, M)$, given in (11), but have to convert that also in terms of $n$ instead of $\varphi$ as the defining variable. This can be done in various ways, but the easiest is to start from (14), omit the inertial contribution of the hot electrons and use $F(n)=\sigma \ln n$, yielding

$$
\begin{aligned}
S_{B}(n, M)= & \frac{n^{2}}{\sigma^{2}}\left\{\frac{M^{2}}{\mu}\left(1-\sqrt{1-\frac{2 \mu \sigma \ln n}{M^{2}}}\right)+(1-f) \sigma(1-n)\right. \\
& +\frac{f}{6}\left(6 M^{2}+2-\left[(M+1)^{2}+2 \sigma \ln n\right]^{3 / 2}\right. \\
& \left.\left.+\left[(M-1)^{2}+2 \sigma \ln n\right]^{3 / 2}\right)\right\} .
\end{aligned}
$$

By assumption, $S_{A}(1, M)=S_{B}(1, M)=S_{A}^{\prime}(1, M)=S_{B}^{\prime}(1, M)$ $=0$, but $S_{A}^{\prime \prime}(1, M) \leq 0$ and $S_{B}^{\prime \prime}(1, M) \leq 0$ are required for the undisturbed conditions to be a (local) unstable maximum, at least on one side. ${ }^{24,32-34}$ Here, primes denote derivatives of
$S_{A}(n, M)$ and $S_{B}(n, M)$ with respect to $n$. The convexity condition becomes

$$
S_{A}^{\prime \prime}(1, M)=\left(\frac{\mu}{M^{2}}+\frac{f}{M^{2}-1}+\frac{1-f}{M^{2}-\sigma}\right) \leq 0 .
$$

When $S_{A}^{\prime \prime}(1, M)=0$, this gives a biquadratic in $M^{2}$, for which one can prove that there are two positive roots, one being smaller, the other larger than 1. This is illustrated in Fig. 1. Therefore, the root obtained for $M^{2}>1$ is the appropriate true normalized electron-acoustic speed in the plasma under discussion, $M_{s}$. Hence, because the normalization cancels out, $M / M_{s}$ represents the true Mach number for the given plasma composition and soliton speed. In physical terms, $M^{2} \geq M_{s}^{2}$ means that the nonlinear structures are (super)acoustic. Single nonzero roots of $S(n, M)$ give positive or negative potential solitons, whereas for double roots $n$ changes from one value at $x=-\infty$ to another at $x=+\infty$, typical for potential kinks (double layers). The full expression for $M_{s}$ involves square roots of terms which are themselves square roots, and have not been reproduced here, as they are cumbersome and not enlightening.

As $M$ is increased above $M_{s}$ for a given plasma, solitons of increasing amplitude are found, until one hits an upper limit in $M$, beyond which solitons do not exist. The upper limit in $M$ may occur because the perturbed density for a specific species vanishes, tends to infinity or becomes complex. That may be when the soliton speed reaches the sonic point. $^{4,19}$ Alternatively, the cutoff can be due to the occurrence of a double root of the Sagdeev potential (signifying a double layer). ${ }^{35}$ Finally, we need to be aware of the limitations imposed by the model, which themselves may give rise to an upper cut-off in speed, as we have noted above $(1<M<\sqrt{\sigma})$.

Returning to consideration of the Sagdeev potential, we know that the sign of $S_{A}^{\prime \prime \prime}\left(1, M_{s}\right)$ determines the polarity of the KdV-like solutions. ${ }^{24,32-34}$ However, we note that these might have large amplitudes, well beyond typical $\mathrm{KdV}$ theory limits, if $M$ can be increased sufficiently above $M_{s}$. If the compositional parameters allow for $S_{A}^{\prime \prime \prime}\left(1, M_{s}\right)$ to go

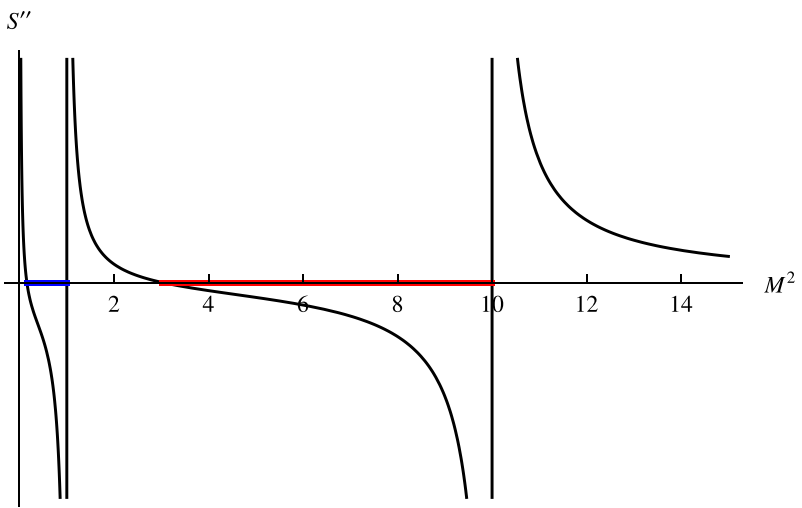

FIG. 1. Qualitative plot of $S_{A}^{\prime \prime}(1, M)$. This has been calculated for $\mu=1 / 20$, $f=0.2$, and $\sigma=10$, values chosen for reasons of graphical clarity, so as not to completely compress the first (ion-acoustic) range in comparison with the second (electron-acoustic) soliton range. These ranges indicate where (16) is obeyed. For other parameter values, the graphs are all topologically equivalent. 


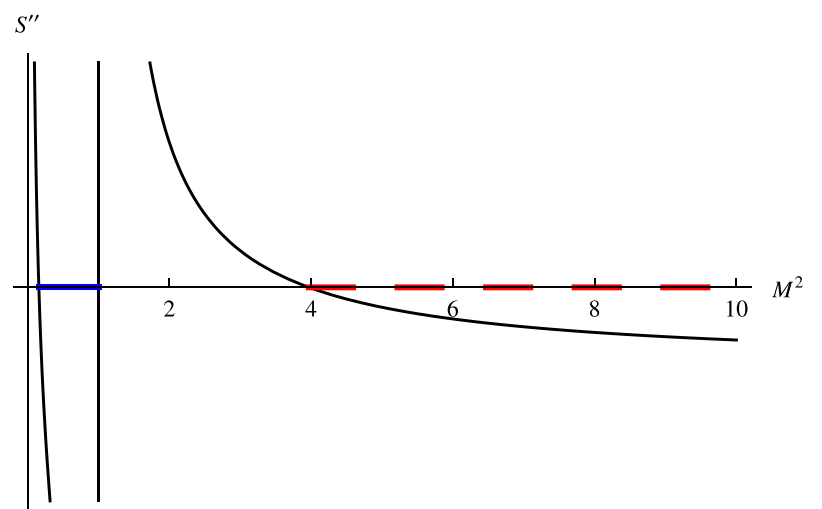

FIG. 2. Qualitative plot of $S_{B}^{\prime \prime}(1, M)$, using the same parameter values and conditions as in Fig. 1, now based on (17). However, the condition $M^{2} \ll \sigma$ severely curtails the electron-acoustic range, which is therefore indicated by a dashed line.

through zero and change sign, the polarity of the KdV-like modes changes. ${ }^{32,36,37}$ This will be the case here, as long as hot electron inertia is retained, but becomes impossible when one adheres to the Boltzmann description of the hot electrons.

Indeed, for the Boltzmann case, the Sagdeev pseudopotential (15) leads to a modification of (16), resulting in

$$
S_{B}^{\prime \prime}(1, M)=\left(\frac{\mu}{M^{2}}+\frac{f}{M^{2}-1}-\frac{1-f}{\sigma}\right) \leq 0,
$$

which is illustrated in Fig. 2. Consequently, a different $M_{S}$ is obtained, with the result that now $S_{B}^{\prime \prime \prime}\left(1, M_{S}\right)<0$ for all $f$. Thus, solitons have the same polarity for all $f$. Specifically, this means that compressive (positive polarity) electronacoustic solitons cannot be found in the Boltzmann paradigm, as is well-known. ${ }^{28,38}$

Examples of $S_{A}^{\prime \prime \prime}\left(1, M_{s}\right)$ and $S_{B}^{\prime \prime \prime}\left(1, M_{s}\right)$ are shown in Fig. 3 for $\mu=1 / 1836$ and $\sigma=100$. We have checked that, over a very wide range in $\mu$ and/or in $\sigma$, the curves shown in Fig. 3 hardly change. In particular, this applies to the polarity switchover point near $f=0.6$ in the case of hot electron inertia. Thus, it is a general characteristic that only negative potential (rarefactive) electron-acoustic solitons occur for a

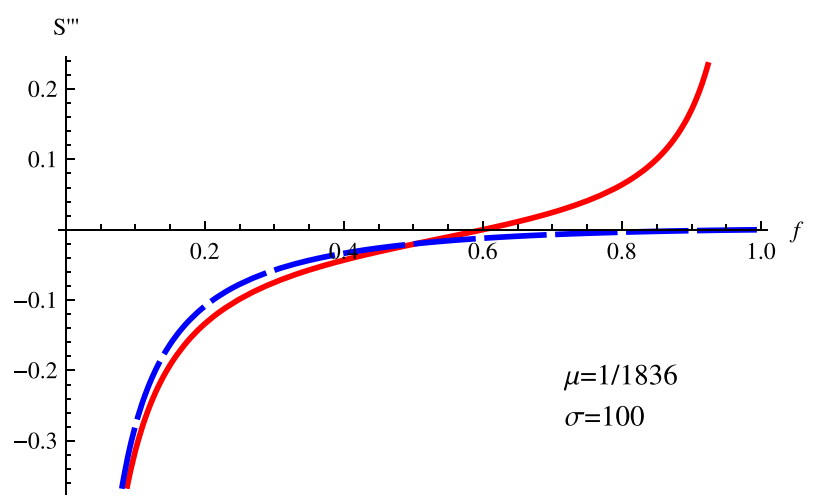

FIG. 3. Plot of $S^{\prime \prime \prime}\left(1, M_{s}\right)$ for $\mu=1 / 1836$ and $\sigma=100$, when hot electron inertia has been included (red solid curve) or neglected (blue dashed curve). It is seen that hot electron inertia allows a polarity change from negative to positive around $f=0.6$, whereas the Boltzmann assumption does not allow for such a polarity change. cool electron density fraction $n_{c 0} / n_{i 0} \lesssim 0.6$, while for higher relative cool electron densities, the solitons are compressive (positive polarity).

A general remark in this and the following is that, while we are able to plot graphs from very near $f=0$ to $f=1$, the regions of $f$ much below 0.2 or above 0.8 are not really credible, for reasons of model limitations (including possible Landau damping in the linear kinetic picture, ${ }^{39,40}$ which would lead to nonlinear waves being unsustainable) and numerical accuracy.

\section{ANALYSIS AND DISCUSSION}

\section{A. Existence domains}

In the numerical interpretation of the existence domains, we will assume that the cold ions are protons, so that $\mu=1 / 1836$. As we will see, varying $\sigma$ over a wide range does not qualitatively change the graphs clarifying the limits on soliton and double layer speeds and amplitudes. We therefore restrict the graphs to two specific values of $\sigma$, namely, 10 and 100, giving hot electron thermal velocities as
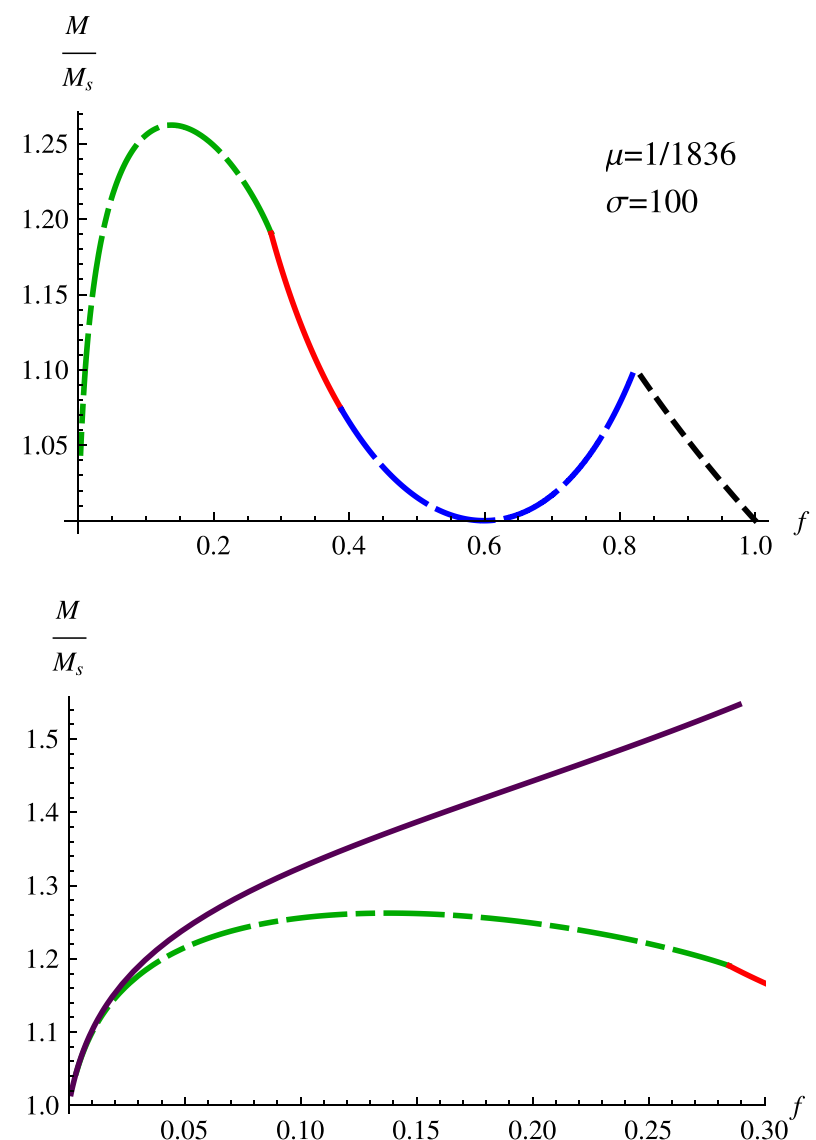

FIG. 4. Existence diagram in Mach number space vs. cool electron density ratio, for $\mu=1 / 1836$ and $\sigma=100$. Upper panel: Soliton velocities are limited, in ascending order, respectively, by cool electron sonic point (green dotted-dashed curve), hot electron sonic point (red solid curve), negative and positive double layers (blue dashed curve), and model limitations (black dotted line), when hot electron inertia is taken into account. Lower panel: Lower $f$ part of the upper panel, with the hot Boltzmann electron limitations also included (purple solid curve). The purple solid curve is drawn up to $M=\sqrt{\sigma}$, although by then the Boltzmann assumption has lost all validity. 
$\sqrt{\sigma}=3.16$ and 10 , and start the discussion with $\sigma=100$. In much of the literature, existence domains are written in terms of a soliton speed $M$ that is normalized relative to an arbitrary fixed speed. However, we have here preferred to use the parameter $M / M_{s}$, the true Mach number, which is more fundamental in terms of physical understanding, but obviously varies with the plasma characteristics, e.g., $f$.

The existence domain for the case when hot electron inertia is taken into account is shown in the upper panel of Fig. 4. It is seen that, for $f$ increasing from 0 to 1 , the soliton velocities are limited by, respectively, the cool electron sonic point $^{4,19}$ (green dotted-dashed curve), the hot electron sonic point (red solid curve), the occurrence of negative and positive double layers (blue dashed curve), and the model limitations (black dotted line). The model limit, where the Mach number equals the hot electron thermal speed, applies for $f \geq 0.822$. The above sequence of upper limits is qualitatively the same as set out in Cattaert et al. ${ }^{17}$

The lower $f$ part of the upper panel is shown in more detail in the lower panel. This panel now also includes the cool electron limitation for the case when the hot electrons are Boltzmann-distributed (purple solid curve), i.e., hot electron inertia is neglected. Surprisingly, the two limit curves coincide only for $f \leqslant 0.05$, and then deviate sharply from one another as $f$ is increased. The purple solid curve is drawn up to $M=\sqrt{\sigma}$, although by then the Boltzmann assumption of an infinite thermal speed has lost all validity. This cutoff occurs at $f=0.289$. The $f$ range is thus severely curtailed, and no changes of polarity are possible. We emphasize that this restriction of the range of applicability of the Boltzmann
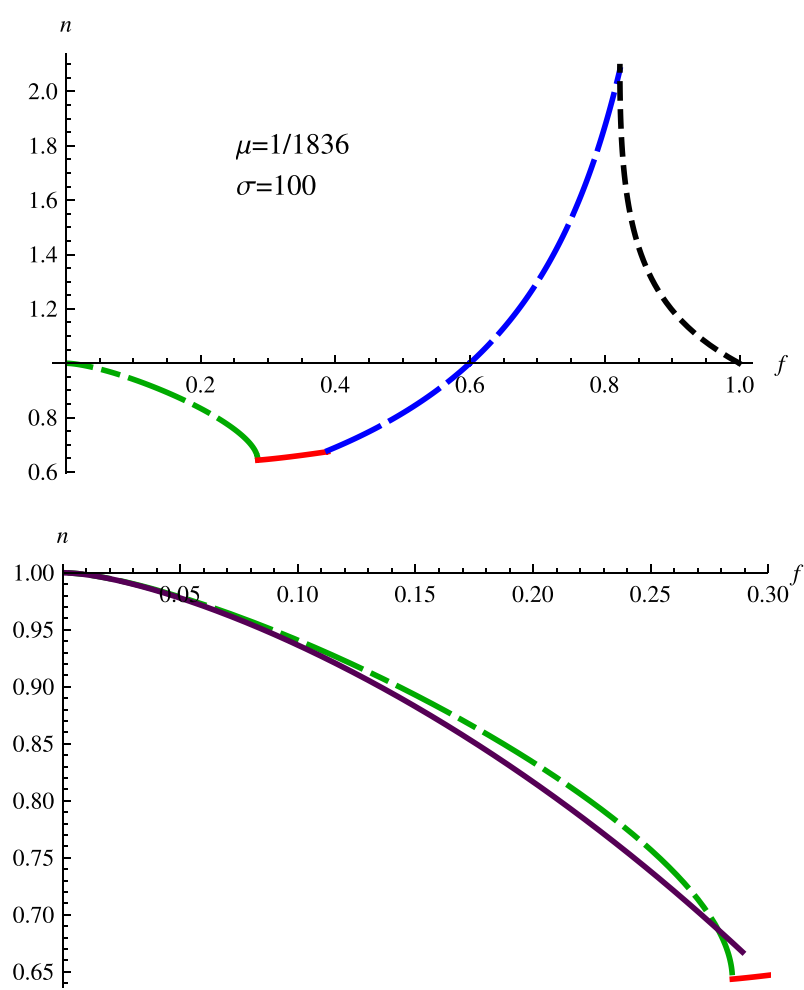

FIG. 5. Existence diagram in amplitude space vs. cool electron density ratio, for $\mu=1 / 1836$ and $\sigma=100$. The curve coding and meaning is as in Fig. 4.
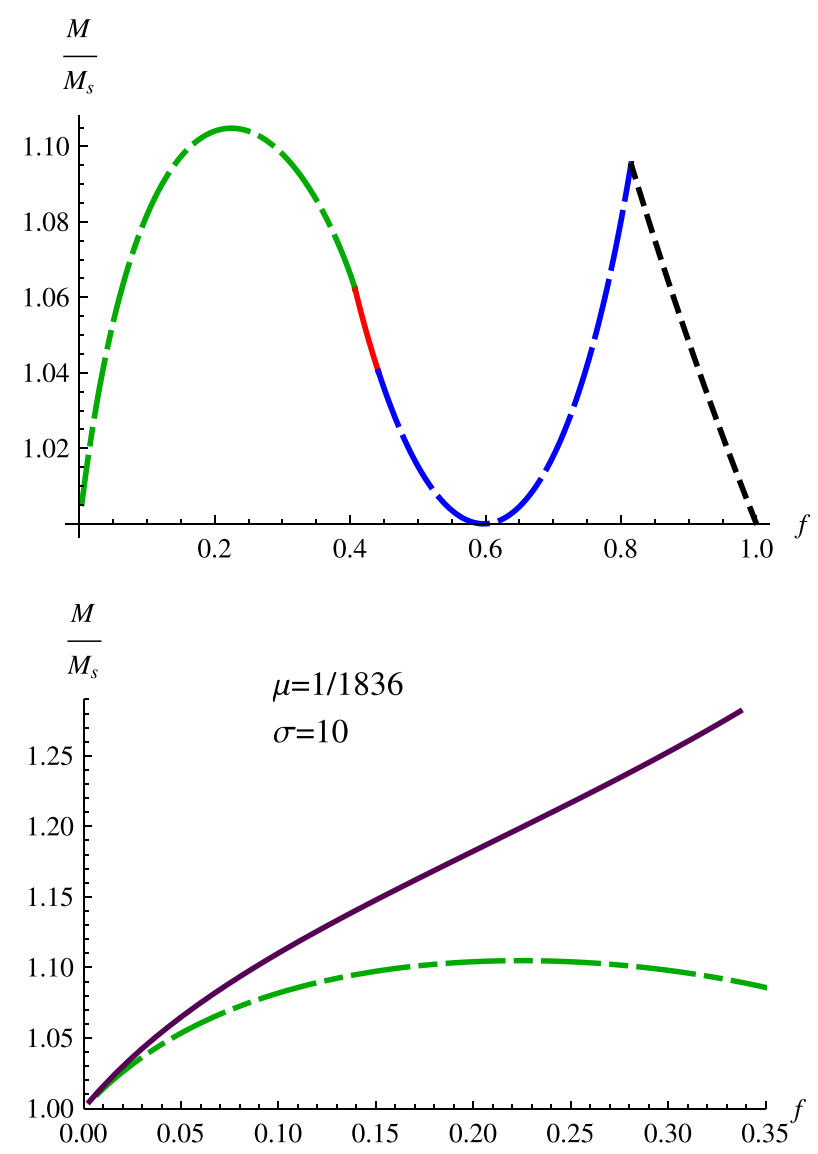

FIG. 6. Existence diagram in Mach number space vs. cool electron density ratio, for $\mu=1 / 1836$ and $\sigma=10$. The curve coding and meaning is as in Fig. 4.
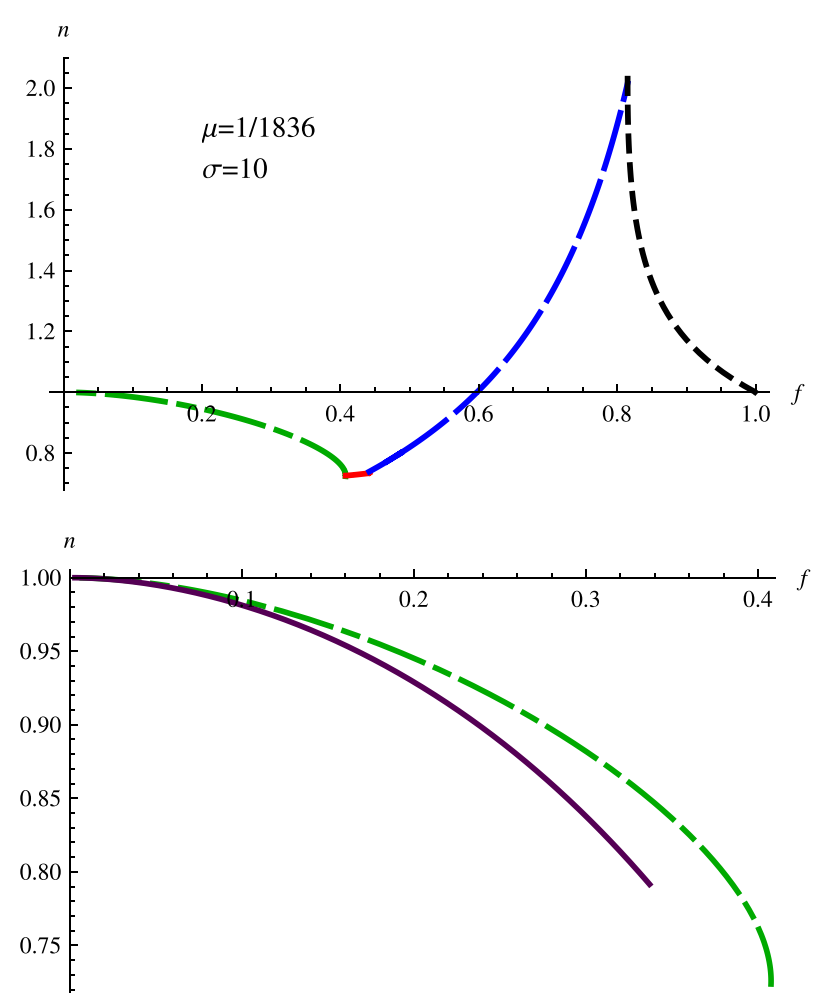

FIG. 7. Existence diagram in amplitude space vs. cool electron density ratio, for $\mu=1 / 1836$ and $\sigma=10$. The curve coding and meaning is as in Fig. 4. 
approximation is recognizable only when finite inertia is considered, and it is thus invariably ignored.

There is also in principle a limit due to infinite cold ion compression, but that is not encountered in practice. The nature of the infinite compression or sonic point constraints is such that points on the limit curves do not represent proper soliton solutions, but indicate how high one can go in $M / M_{s}$. The double layers, on the other hand, represent acceptable solutions, and signal the end of a soliton range, ${ }^{35}$ at given $f$, as $M / M_{s}$ is increased. We note that double layers are not found when the hot electrons have a Boltzmann distribution. This result agrees with, for instance, earlier work, ${ }^{41}$ which showed that weak electron-acoustic double layers do not occur, as well as a more recent study of arbitrary amplitude structures in a fully adiabatic plasma. ${ }^{28}$

We now represent the analogous information in terms of the hot electron density vs. $f$, in Fig. 5. It will be recalled that the equilibrium hot electron density is given by $n=1$. For clarification, we point out that for given $f$, as increasing soliton speed $M$ is considered, soliton amplitudes increase from $M=M_{s}$ to the limit curve shown. It is seen that in the range $f=0$ to 0.599 , the solitons and double layers are rarefactive in the hot electron density. Using (7), one then obtains negative electrostatic potentials, $\varphi<0$, while (5) and (6) show that the soliton is also rarefactive in the ion density, but compressive in the cool electron density. We will call such
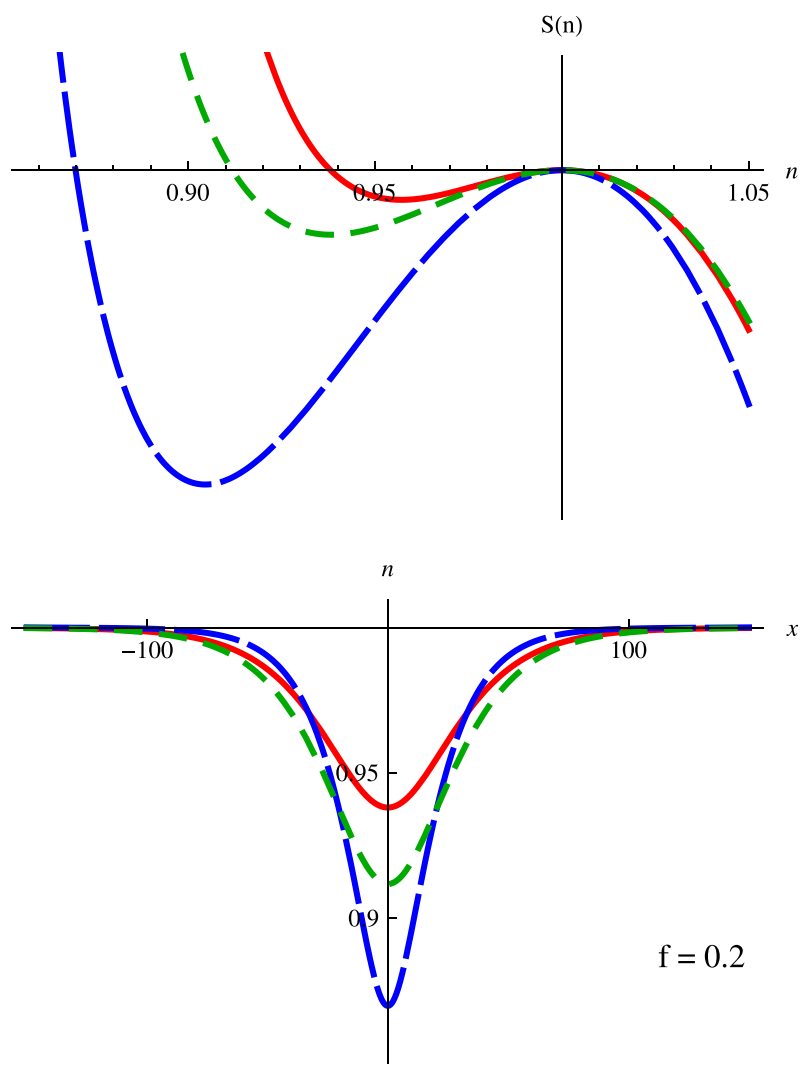

FIG. 8. Two examples of Sagdeev pseudopotentials (14) for $\mu=1 / 1836$, $\sigma=100$, and $f=0.2$ (upper panel), with the respective soliton profiles (lower panel). The red solid curve is for $M / M_{s}=1.1$ and the blue dashed curve for $M / M_{s}=1.2$. For comparison, the green dotted curves are for the Boltzmann description of the hot electrons, given in (15), for $M / M_{s}=1.2$. In this $f$ range, all solitons are ultimately limited for increasing $M / M_{s}$ by cool electron sonic points, which, however, do not themselves give acceptable solutions. solutions "rarefactive" for short, but point out that in plasmas with more than two species the labels rarefactive and compressive are ambiguous. In the same vein, from $f=0.599$ the solitons and double layers are "compressive," with $\varphi>0$. It is obvious that without hot electron inertia only rarefactive or dark solitons are possible $(\varphi<0)$. That is, omitting hot electron inertia means that compressive or bright solitons cannot occur. However, in the range from $f=0$ to 0.3 , there is seemingly little difference in amplitude between the Boltzmann and inertial cases, as indicated in the lower panel of Fig. 5, a point to which we shall return in Sec. III B.

In Figs. 6 and 7, information is given for $\mu=1 / 1836$ and $\sigma=10$, in the same coding as in Figs. 4 and 5 , respectively. The comparison between the figures for $\sigma=100$ and $\sigma=10$ shows that qualitatively the conclusions are similar, and although there are some quantitative differences, the maximum amplitudes are hardly affected by the lower temperature ratio. We do note, however, that for $\sigma=10$ the ranges in $f$ in which the cool and the hot electron sonic points provide the amplitude limits, are wider and narrower, respectively, than is the case for $\sigma=100$.

Figures 4 and 6 are reminiscent of results obtained by Cattaert et al. ${ }^{17}$ for a rather simpler configuration: the ions were treated as an immobile neutralizing background (in an inertial frame) and the cool electrons treated as completely cold. This omitted all ion dynamics and all cool electron thermal effects, but captured the gist of what happens when
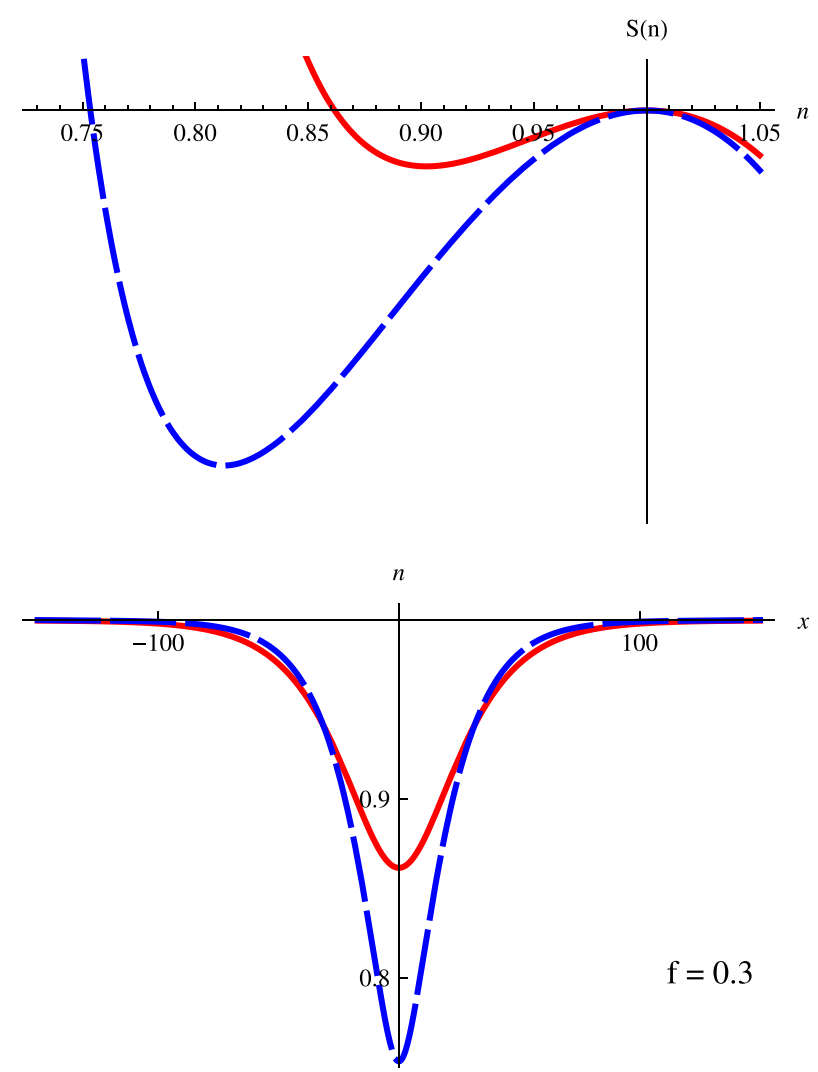

FIG. 9. Two examples of Sagdeev pseudopotentials (14) for $\mu=1 / 1836$, $\sigma=100$, and $f=0.3$ (upper panel), with the respective soliton profiles (lower panel). The red solid curve is for $M / M_{s}=1.1$ and the blue dashed curve for $M / M_{s}=1.15$. Here, the solitons are ultimately limited by hot electron sonic points, which, however, do not themselves give acceptable solutions. 
inertia is retained for the hot electrons. The normalization had to be done in terms of the hot electron parameters, with essentially $\mu=0$ and $0<M<1$, whereas in the present paper it is done as functions of the cool electron (thermal) parameters, purely for analytic expediency. Also, in the earlier study, ${ }^{17} M^{2}$ was plotted as a function of $f$ in Figs. 2, 8, and 9 , whereas we now use the true Mach number $M / M_{s}$.

More recently, Maharaj et $a l^{28}$ obtained the same qualitative sequence when considering adiabatic hot electrons, albeit the switch in polarity was found at a much lower value of $f$, namely, at $f=0.43$. Interestingly, Cattaert et al. ${ }^{17}$ considered a number of different values of $\gamma$ for the hot electrons and found that the results were robust in the qualitative behaviour, with the actual value of $f$ at which the polarity changed varying from $f \simeq 0.43$ for $\gamma=3$ to $f \simeq 0.6$ for $\gamma=1$.

\section{B. Pseudopotentials, solitons, and double layers}

In the figures which follow, we will illustrate some Sagdeev pseudopotentials in the upper panels, using (14) or, where appropriate, (15), while their corresponding soliton or double layer solutions are shown in the lower panels. We again consider the case $\mu=1 / 1836$ and $\sigma=100$, and various values of $f$.

We start the discussion with $f=0.2$ in Fig. 8 , in the range where all solitons are ultimately limited (for increasing $M\left(M_{s}\right)$ by cool electron sonic points, in this case at a value
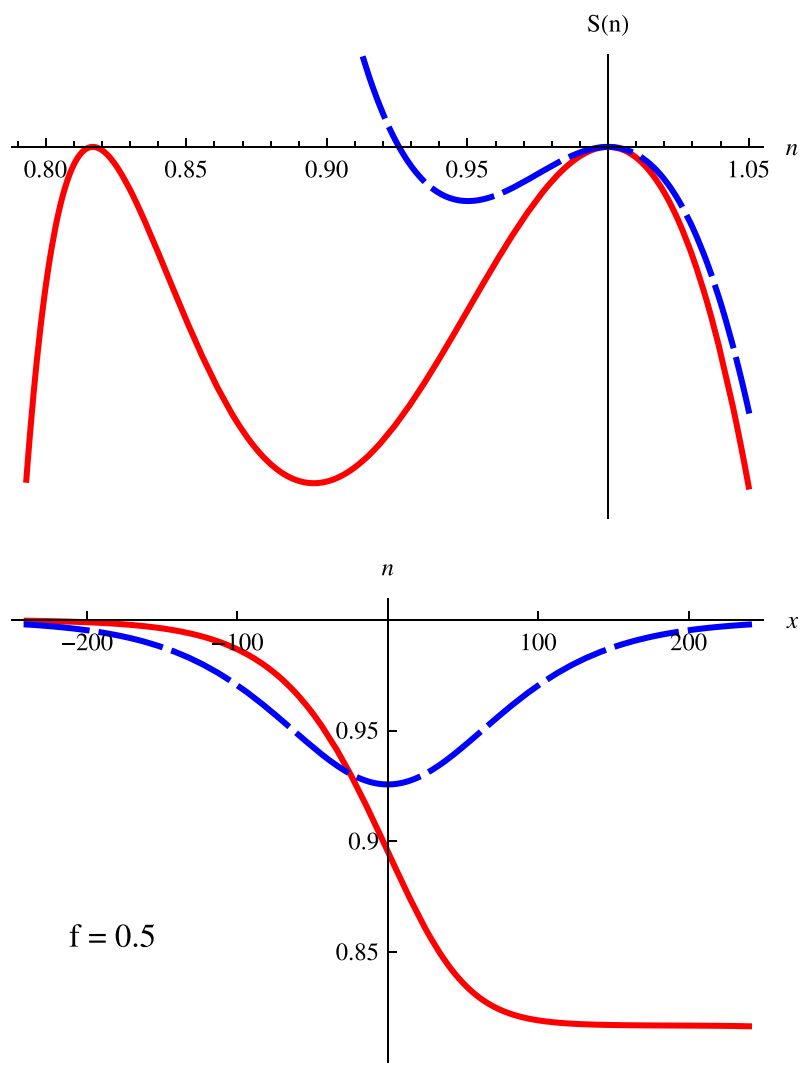

FIG. 10. Two examples of Sagdeev pseudopotentials (14) for $\mu=1 / 1836$, $\sigma=100$, and $f=0.5$ (upper panel), with the respective soliton or double layer profiles (lower panel). The red solid curve is for $M / M_{s}=1.016$ and the blue dashed curve for $M / M_{s}=1.01$. In this range, the solitons are ultimately limited by negative double layers, which are perfectly acceptable solutions, as illustrated by the red solid curve.
1.25. However, the latter do not themselves yield acceptable solutions, by nature of the stagnation effect occurring there. Here, the red solid curves are for $M / M_{s}=1.1$, the blue dashed curves for $M / M_{s}=1.2$. For comparison, the green dotted curves are for the Boltzmann description of the hot electrons, given in (15), for $M / M_{s}=1.2$. It is seen that an increase in the soliton speed, through the Mach number $M / M_{s}$, increases the amplitude in absolute value, but restricts the width. In fact, using (13), it is clear from the far greater well-depth found from $S_{A}(n)$ for $M / M_{S}=1.2$, that the associated soliton profile has a far steeper slope than is the case for $M / M_{s}=1.1$. At the same Mach number, the Boltzmann profiles present a lower amplitude, but this effect is related in part to the different definition of the acoustic speed $M_{s}$, as may be seen from a comparison of (16) and (17).

Similar tendencies will be obvious for higher values of $f$, as illustrated in Fig. 9 for $f=0.3$, where the limiting factor is the hot electron sonic point. It is evident that for Boltzmann hot electrons there cannot be a sonic point, and hence comparison between the solutions of (14) and (15) is meaningless. In addition, one would be in the regime where $M$ exceeds $\sqrt{\sigma}$ if one were to take proper account of the hot electron inertia.

Next come the double layer limitations, which represent perfectly acceptable solutions of the nonlinear propagation problem. This is illustrated in Fig. 10 for $f=0.5$, with
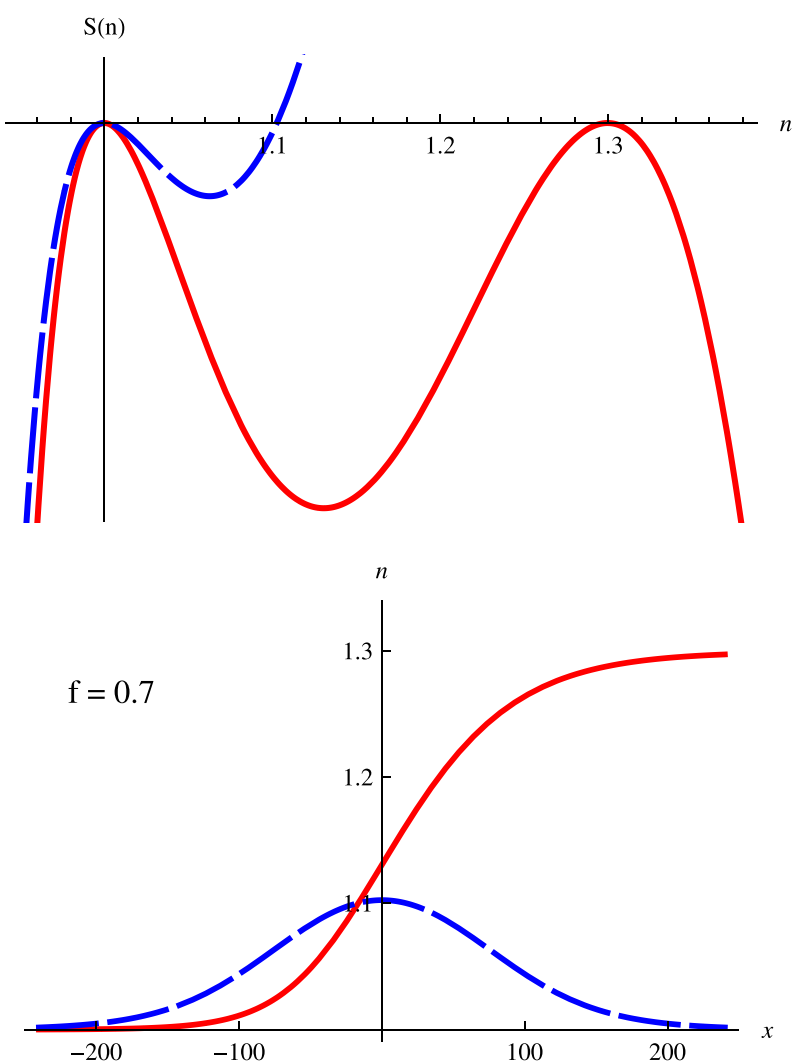

FIG. 11. Two examples of Sagdeev pseudopotentials (14) for $\mu=1 / 1836$, $\sigma=100$, and $f=0.7$ (upper panel), with the respective soliton or double layer profiles (lower panel). The red solid curve is for $M / M_{s}=1.017$ and the blue dashed curve for $M / M_{s}=1.01$. In this range, the solitons are ultimately limited by positive double layers, which are perfectly acceptable solutions, as illustrated by the red solid curve. 
negative solitons and double layers, and in Fig. 11 for $f=0.7$, now for positive solitons and double layers, on opposite sides of the polarity change at $f=0.599$.

Traditionally, double layers are defined to be made up of adjacent layers of opposite charge density polarity. From Poisson's equation, the resulting two opposing curvatures in electrostatic potential lead to a kink in the potential. They are identified numerically by double roots, in electrostatic potential $\varphi$, of the Sagdeev pseudopotential $S(\varphi, M)$. In what has gone before, we have identified double roots, in hot electron density $n$, of $S(n, M)$ as double layers. To show that the soliton profiles illustrated in the lower panels of Figs. 10 and 11, in particular, the double layers, indeed correspond to what is usually understood by the concept, we convert the information to $\varphi$ with help of the relation (7). As Fig. 12 shows, the double layers in $n$ correspond exactly to those in $\varphi$, but, of course, on different scales for the amplitudes. The fairly large values for the double layer amplitudes (in absolute value) are here a corollary of our normalization in terms of the cool electron thermal velocity and temperature, rather than, say, the hot electron variables or a weighted average.

Finally, we point out that we have chosen not to go much higher than $f=0.7$, because the model limitations, represented in Figs. $4-7$ by black dotted lines beyond $f=0.822$, are expressed by $M=\sqrt{\sigma}$, which in principle cannot be accessed, as the structure velocity would equal the hot electron thermal velocity!
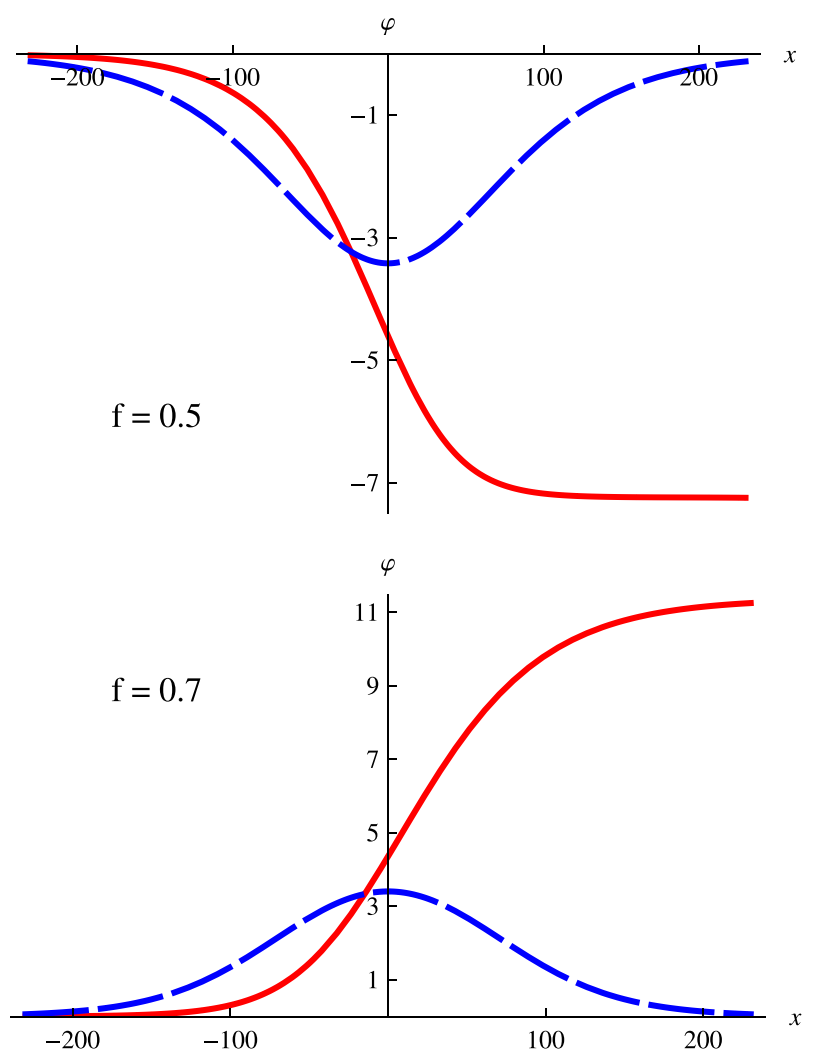

FIG. 12. The soliton profiles illustrated in the lower panels of Figs. 10 and 11 , in particular, the double layers in $n$, correspond exactly to those in $\varphi$, but, of course, on different scales for the amplitudes.

\section{CONCLUSIONS}

We have investigated the propagation of arbitrarily large electron-acoustic solitons in a plasma with cold positive ions, cool adiabatic and hot isothermal electrons, with the inclusion of full inertial effects for all species. This plasma model is appropriate for the study of the standard electronacoustic wave, the phase speed of which is bracketed by the two thermal speeds, $v_{t c} \ll v_{p h} \ll v_{t h}$. It follows that the hot electrons can thermalise ahead of the wave and are isothermal, while the cool electrons are effectively insulated and hence behave adiabatically. Furthermore, one can then easily investigate the effects of ignoring the hot electron inertia, as the commonly assumed Boltzmann relation is the inertialess limit of the isothermal form.

To study the inertial isothermal case, the Sagdeev pseudopotential $S(n, M)$ has to be expressed in terms of the hot electron density, rather than the more routine electrostatic potential $\varphi$, to obtain a completely analytical expression. It is then easy to switch the hot electron inertia off, to compare the new results with those for an inertialess hot component.

Unlike the inertialess Boltzmann case, the existence domains for Mach numbers $M / M_{s}$ and hot electron densities $n$ clearly show that both rarefactive and compressive solitons can exist, if the range of $f$ goes beyond the point where $S^{\prime \prime \prime}\left(1, M_{s}\right)$ changes sign. For small $f$, the hot electrons and the cold ions are rarefied, but the cool electrons are compressed, which corresponds to $\varphi<0$. Density limitations, giving rise to upper limits in $M$, come first from the cool electron sonic point, and then the hot electron sonic point, until a range of rarefactive double layers is the limiting factor. Increasing $f$ further yields a switch in soliton character to compressive double layers, corresponding to $\varphi>0$, and meaning that the ions and hot electrons are compressed, the cool electrons rarefied. The end is reached when $M=\sqrt{\sigma}$ represents the breakdown of the model assumptions. These qualitative results are but little influenced by variations in $\mu$ and $\sigma$.

The fully inertial isothermal case immediately reduces to a Boltzmann form when the inertia is neglected. A comparison with the inertialess case for the hot electrons shows various aspects. To begin with, there is, of course, only the cool electron sonic point limitation, which could at first sight go on for all $f<1$. However, we now have a limit on $M$, namely, $M=\sqrt{\sigma}$, beyond which the Boltzmann results for the hot electrons lose credibility. We note that this point of considering the limitations of the model is sometimes neglected. The model limit occurs already at around $f=0.3$, where, compared to the fully inertial case, the discrepancy in the maximum allowed value of $M / M_{s}$ is already significant, although the estimates for the maximum densities at given $f$ are quite close, and certainly seem to indicate the right order of magnitude. What cannot be reproduced at all by the Boltzmann distribution are the double layer solutions, and the switch from rarefactive to compressive form or negative to positive polarity ( $\operatorname{sign}$ of $\varphi$ ).

The results for inertial isothermal hot electrons agree with those of Cattaert et al. ${ }^{17}$ who used the gas-dynamic technique, and made some slightly different assumptions regarding the ions and cool electrons. Similarly, there is 
qualitative agreement with the work of Maharaj et al., ${ }^{28}$ who assumed adiabatic hot electrons and relatively hot adiabatic ions. However, quantitative details, e.g., the cool electron density fraction at which the soliton polarity is reversed, appear to be dependent essentially on the value of $\gamma$ assumed for the hot electrons, with the other two species playing only minor roles.

\section{ACKNOWLEDGMENTS}

Useful discussions with Richard Mace are gratefully acknowledged. M.A.H. thanks the National Research Foundation of South Africa for partial support under Grant No. 68911. Any opinions, findings, and conclusions expressed herein are those of the authors, and the NRF accepts no liability whatsoever in this regard.

${ }^{1}$ D. J. Korteweg and G. de Vries, Philos. Mag. 39, 422 (1895).

${ }^{2}$ H. Washimi and T. Taniuti, Phys. Rev. Lett. 17, 996 (1966).

${ }^{3}$ R. Z. Sagdeev, in Reviews of Plasma Physics, edited by M. A. Leontovich (Consultants Bureau, New York, 1966), Vol. 4, p. 23.

${ }^{4}$ F. Verheest, T. Cattaert, G. S. Lakhina, and S. V. Singh, J. Plasma Phys. 70, 237 (2004).

${ }^{5}$ F. Verheest, M. A. Hellberg, and G. S. Lakhina, Astrophys. Space Sci. Trans. 3, 15 (2007).

${ }^{6}$ R. A. Cairns, A. A. Mamun, R. Bingham, R. Boström, R. O. Dendy, C. M. C. Nairn, and P. K. Shukla, Geophys. Res. Lett. 22, 2709, doi:10.1029/ 95GL02781 (1995).

${ }^{7}$ V. M. Vasyliunas, J. Geophys. Res. 73, 2839, doi:10.1029/ JA073i009p02839 (1968).

${ }^{8}$ M. A. Hellberg, R. L. Mace, T. K. Baluku, I. Kourakis, and N. S. Saini, Phys. Plasmas 16, 094701 (2009).

${ }^{9}$ C. Tsallis, J. Stat. Phys. 52, 479 (1988).

${ }^{10}$ J. A. S. Lima, R. Silva, Jr., and J. Santos, Phys. Rev. E 61, 3260 (2000).

${ }^{11}$ H. H. Kuehl and C. Y. Zhang, Phys. Fluids B 3, 26 (1991).

${ }^{12}$ S. Ghosh, S. Sarkar, M. Khan, and M. R. Gupta, Planet. Space Sci. 48, 609 (2000).

${ }^{13}$ B. Sen, B. Das, and P. Chatterjee, Eur. Phys. J. D 49, 211 (2008).

${ }^{14}$ S. N. Barman and A. Talukdar, Astrophys. Space Sci. 334, 345 (2011).
${ }^{15}$ W. K. M. Rice, M. A. Hellberg, R. L. Mace, and S. Baboolal, Phys. Lett. A 174, 416 (1993).

${ }^{16}$ R. L. Mace and M. A. Hellberg, Planet. Space Sci. 41, 235 (1993).

${ }^{17}$ T. Cattaert, F. Verheest, and M. A. Hellberg, Phys. Plasmas 12, 042901 (2005).

${ }^{18}$ F. Verheest, T. Cattaert, and M. A. Hellberg, Space Sci. Rev. 121, 299 (2005).

${ }^{19}$ J. F. McKenzie, Phys. Plasmas 9, 800 (2002).

${ }^{20}$ M. Berthomier, R. Pottelette, and R. A. Treumann, Phys. Plasmas 6, 467 (1999).

${ }^{21}$ R. L. Mace and M. A. Hellberg, Phys. Plasmas 8, 2649 (2001)

${ }^{22}$ J. F. McKenzie, J. Plasma Phys. 69, 199 (2003).

${ }^{23}$ F. Verheest, Phys. Plasmas 13, 082301 (2006).

${ }^{24}$ T. K. Baluku and M. A. Hellberg, Plasma Phys. Controlled Fusion 53, 095007 (2011)

${ }^{25}$ G. S. Lakhina, A. P. Kakad, S. V. Singh, and F. Verheest, Phys. Plasmas 15, 062903 (2008).

${ }^{26}$ F. Verheest and M. A. Hellberg, J. Plasma Phys. 76, 277 (2010).

${ }^{27}$ S. K. Maharaj, R. Bharuthram, S. V. Singh, and G. S. Lakhina, Phys. Plasmas 19, 072320 (2012).

${ }^{28}$ S. K. Maharaj, R. Bharuthram, S. V. Singh, and G. S. Lakhina, Phys. Plasmas 19, 122301 (2012).

${ }^{29}$ S. S. Ghosh, K. K. Ghosh, and A. N. Sekar Iyengar, Phys. Plasmas 3, 3939 (1996).

${ }^{30}$ F. Verheest, M. A. Hellberg, and I. Kourakis, Phys. Plasmas 15, 112309 (2008).

${ }^{31}$ F. Verheest, G. S. Lakhina, and M. A. Hellberg, Phys. Plasmas 21, 062303 (2014).

${ }^{32}$ T. K. Baluku, M. A. Hellberg, I. Kourakis, and N. S. Saini, Phys. Plasmas 17, 053702 (2010).

${ }^{33}$ T. K. Baluku, M. A. Hellberg, and F. Verheest, Europhys. Lett. 91, 15001 (2010).

${ }^{34}$ F. Verheest and M. A. Hellberg, Phys. Plasmas 17, 102312 (2010).

${ }^{35}$ S. Baboolal, R. Bharuthram, and M. A. Hellberg, J. Plasma Phys. 44, 1 (1990).

${ }^{36}$ F. Verheest, Phys. Plasmas 18, 083701 (2011).

${ }^{37}$ F. Verheest, M. A. Hellberg, and T. K. Baluku, Phys. Plasmas 19, 032305 (2012).

${ }^{38}$ R. L. Mace, S. Baboolal, R. Bharuthram, and M. A. Hellberg, J. Plasma Phys. 45, 323 (1991).

${ }^{39}$ S. P. Gary and R. L. Tokar, Phys. Fluids 28, 2439 (1985).

${ }^{40}$ R. L. Mace and M. A. Hellberg, J. Plasma Phys. 43, 239 (1990).

${ }^{41}$ R. L. Mace and M. A. Hellberg, J. Plasma Phys. 49, 283 (1993). 